\title{
A Credit-Based System for Traffic Routing in Support of Vehicular Networks
}

\author{
Ammar Kamel, Computer Department, College of Medicine, Al-Mustansiriyah University, Iraq \\ iD https://orcid.org/0000-0002-3437-3388 \\ Maysaa Husam, Computer Department, College of Medicine, Al-Mustansiriyah University, Iraq \\ Zaid Shafeeq Bakr, College of Information Engineering, Al-Nahrain University, Iraq \\ Ziad M. Abood, College of Education, Al-Mustansiriyah University, Iraq
}

\begin{abstract}
Network routing has a great impact on the efficiency and reliability of the traffic network system in a real-world scenario. To date, achieving network-consistent performance is the main goal of many traffic network research studies. In this research, a mixed strategy game-theory model for network routing is proposed that discovers the optimal strategies that can be adopted by network route players in a network graph. This model has been validated by measuring the model outcomes using quantal response equilibrium (QRE) technique, which explores the players' noisy decisions by comparing the utilized optimal strategies with Nash equilibrium. The experimental results demonstrate that there is an equilibrium with a mixed strategy of a given network.
\end{abstract}

\section{KEYWORDS}

Game Theory, Nash Equilibrium, Quantal Response Equilibrium (QRE), Traffic Routing

\section{INTRODUCTION}

The modern world is full of networks as they impact our lives; the traffic road network is one prominent example. In the last few years there has been considerable interest in the network traffic routing management.

Generally speaking, every network user wishes to have the best service possible at the lowest possible cost. Regulation of traffic in many domains cannot impose such as in "The internet or road networks", we are concerned in those settings where each user acts according to his /her own selfish interests. Therefore, the networks participants' actual behavior can be predicted by utilizing the principles of game theory. Exploiting game theory approaches represent one of the most sophisticated solutions to handle various nowadays problems (Allen \& Luiz, 2006).

In the same direction, because of the growing number of vehicles is gradually causing congestion issue, routing researches nowadays are moving toward avoiding network-level routing inadequacies by imposing adapters (users) either to utilize routes themselves or to adapt overlay routing networks. It was possible to alleviate routing management which direct route adapters to routes with few traffic flows to gain the shortest travel time. However, the latest route management algorithms pay more attention to one adapter and neglect the adapter coordination which results in significant deterioration of efficiency caused by lack of coordination (D. \& J., Reasoning about a highly connected world, 2010). 
We suppose that every user will always choose the minimum latency path to its destination and also all users are rational and non-malicious. All these can be viewed as a non-cooperative game where each user plays the best response given the state of all other users, and thus we expect the routes chosen to form Nash equilibrium. (Hyunmyung, Jun-Seok, \& R, 2009)

Nash equilibrium usually takes place when all the players take the best reaction to the other's strategic choices jointly. That is, despite what the other player is doing, he or she opts for the right move. Of course, this would be easier if the players knew which strategies their opponents would choose on a timely manner. (Ken, 2008)

A variety of approaches and models based on game theory have been proposed with regard to discovering optimal solutions for network reliability "connectivity" and traffic routing problems. The following is a brief illustration of these approaches and models.

In (Mohammed, Ahmed, \& Abdallah, September, 2015), a new automated network routing algorithm has been developed. The developed algorithm replaces the ordinary state-of-the-practice control system by utilizing future automated vehicle capabilities at intersections. The proposed algorithm was inspired by the chicken-game. In the same direction, the study argues that, when Nash's constraints applied, the participated vehicles follow the Nash equilibrium. The extracted outcomes of the simulated experiments showed that the proposed study achieves a $49 \%$ reduction in average travel time delay and an $89 \%$ reduction in overall delay when it is compared to the all-way stop sign-controlled intersection.

A level-k game-theoretic framework for modeling traffic presented in (Nan, Ilya, Anouck, \& Yildiray, June 2018). The proposed framework models the time-extended, multi-step, and interactive decision making of autonomous vehicles (AV) at uncontrolled intersections. Also, a rule-based approach exploited to measure the conditions that lead to conflicts between vehicles at the intersection. It was shown, through simulations that the proposed modeling traffic framework has the capability to resolve conflicts of different driver types at the intersection.

A distributed cooperative routing algorithm DCR is proposed in (J., J., Q., \& B., 2019). The proposed DCR algorithm has been used to overcome misleading coordinate vehicles problem using evolutionary game theory. The proposed method utilized roadside units (RSUs) to evaluates the performance of DCR and measure its effectiveness to balance the traffic flow distribution as well as compute the total travel time. The extracted results show that the total travel time has been reduced to the minimum.

In (B., 2019), a game theory model that utilized particle swarm optimization is presented. The proposed model optimizes the intersections coordination by combining multi-agent coordination technique that measures the queuing length and delay time between the intersections. The study outcomes have shown that the effectiveness of utilizing the proposed coordinated optimal control model to minimize the average vehicle delay and the average queue length of each intersection. Furthermore, for the sake of verifying the control effectiveness, the Vissim simulation software has been utilized.

The study in (L., Y., L., Z., \& C., 2010) presented a Stackelberg game model to minimize the total traffic travel cost in User Equilibrium (UE) traffic assignment problem. A Cournot-Nash game theory model was presented and assigned to equilibrium players as followers whereas the System Optimal (SO) player become a leader for a selected route. The Cournot-Nash and user equilibrium players choose the route provided by the SO player. The SO user, on the other hand, selects the routes to reduce overall travel costs for the traffic system. In term of finding the best outcomes of the proposed model, the Lagrange multiplier method was applied.

According to a study in (Andra, Jozsef, Attila, Gabor, \& Dmitri, 2015) by using game theory, certain real networks with special skeleton are Nash equilibrium that maximized efficiency and minimize the cost of navigation. The purpose of these networks is to find an optimal trade-off between the network cost and navigability. Furthermore, the study showed that these skeletons give the ability to determine the minimal number of network edges that can be changed in order to improve the network navigation efficiency. The proposed game-theoretic approach emphasized on 
Nash equilibrium networks that are minimalistic idealizations. However, error tolerance and policy compliance functions have not utilized in the proposed approach.

The paragraphs below are organized according to the conducted research policy. The first section represents a brief review of the studies that have been accomplished in network traffic routing and protections. The second section gives an illustration of the game theory principles and schemes and presents the most related solution model of network traffic management. The third section presents the proposed model for network traffic routing and protection. Testing and evaluating the constructed results from the proposed model will be described in the section four. Through section five and six, the conclusion and suggestions for the future works will be described.

\section{Principles in Game Theory}

Game theory represents one of the most important solution models that give a new perspective horizon for network traffic management problems (Jing, Wei, \& Kun, 2009). Game theory is the study of strategic engagement. That is, the decision has an effect on the actions of the other player and vice versa. A game scheme is made up of three basic components: a set of players (users, drivers, token station, etc.), a set of actions, and a set of preferences. In a network system, the players (decision makers) are most often the nodes of the network. The actions are flow control parameter, transmission power level, or any other factor that is under control of the node. The action profile can be constructed as a result of the player's chosen actions, and that represents the game outcome. For each player, a preference relationship reflects the evaluation of all potential outcomes by that player.

1. Mixed Strategies: Given a game in strategic form we expand a player's strategy set over his strategies to the set of all probability distributions. The new set's elements are called mixed strategies, while the elements of the original set of strategies are considered pure strategies. A mixed strategy can be defined, then, as a distribution of probabilities over pure strategies.

Definition 1: Let $\mathrm{G}=\left(\mathrm{N},\left(\mathrm{S}_{\mathrm{i}}\right)_{\mathrm{i} \in \mathrm{N}},\left(\mathrm{u}_{\mathrm{i}}\right)_{\mathrm{i} \in \mathrm{N}}\right)$ be a strategic-form game where each player's set of strategies is finite. A mixed strategy of player $\mathrm{i}$ is a probability distribution over his set of strategies $\mathrm{S}_{\mathrm{i}}$. Hence:

$$
\sum_{i}:=\left\{\sigma_{i}: S_{i} \rightarrow[0,1]: \sum_{s_{i} \in S_{i}} \sigma_{i}\left(s_{i}\right)=1\right\}
$$

Is the set of mixed strategies of player $i$. Therefore, the probability distribution over Si: $\sigma_{i}=\left(\sigma_{i}\left(s_{i}\right)\right)_{s_{i} \in S_{i}}$ is a mixed strategy of Player $\mathrm{i}$. The number $\sigma_{\mathrm{i}}\left(\mathrm{s}_{\mathrm{i}}\right)$ is the probability of playing the strategy $s_{i}$. To distinguish between the mixed strategies $\sum_{i}$ and the strategies $S_{i}$, the latter is called pure strategies.

2. Nash Equilibrium: Dominance is an incredibly significant principle in game theory. A player who knows the strategies used by the other players plays a game in which only he is called upon to choose a strategy. Thus, when that player becomes rational, he chooses the best approach to certain strategies that other players utilize. Each player has combinations of moves that called outcomes. An outcome is called a pure Nash equilibrium, provided that by deviating from the move no one can receive a higher payoff while all other players stick to their choices. An acquired 
higher payoff can be possible for a player but only if two or more players change their move promptly. (Prisner, 2014)

Definition 2: A strategy vector $\mathrm{s} *=(\mathrm{s} * 1, \ldots, \mathrm{s} * \mathrm{n})$ is a Nash equilibrium if for each player $\mathrm{i} \in$ $\mathrm{N}$ and each strategy si $\in \mathrm{Si}$ the following is satisfied: ui $(\mathrm{s} *) \geq$ ui $(\mathrm{si}, \mathrm{s} *-\mathrm{i})$. The payoff vector $\mathrm{u}(\mathrm{s} *)$ is the equilibrium payoff corresponding to the Nash equilibrium $\mathrm{s} *$. The strategy $\hat{s_{i}} \in \mathrm{Si}$ is a profitable deviation of player $\mathrm{i}$ at a strategy vector $\mathrm{s} \in \mathrm{S}$ if $\operatorname{ui}\left(\hat{s_{i}}, \mathrm{~s}-\mathrm{i}\right)>\operatorname{ui}(\mathrm{s})$.

Nash equilibrium is a strategy vector at which no player has a competitive variance. The Nash equilibrium is known as equilibrium, and also as a point of equilibrium. As defined above, it illustrated that no player $i$ has a profitable unilateral deviation from $s^{*}$. The Nash equilibrium can be equivalently expressed in terms of the best-reply concept (Michael, Eilon, \& Shmuel, 2013).

3. Bimatrix Game: It is a concurrent game of two players who have a finite number of possible strategies represented in two matrices (A and B), respectively. Hence A and B are $m \times n$ matrices of payoffs of both player 1 and 2. (Noam, Tim, Eva, \& Vijay, Algorithmic game theory, 2007)

\section{MATERIALS AND METHODS}

\section{The Proposed Routing Model (Game Theory Model)}

Figure 1 has the properties: no cooperative, mixed strategies and complete information.

Let's assume that a flow network $\mathrm{G}=(\mathrm{V}, \mathrm{E})$ is a directed graph in which each edge $\mathrm{e} \in \mathrm{E}$ has a nonnegative capacity c (e) $>0$, and each $n \in N$. Every $n$ represents a direct link (connection) between any two nodes. The delay of the path $\mathrm{P}=(\mathrm{vi}, \ldots, \mathrm{vd})$ can be computed as: wi $=\sum_{e i j \in P} c i j$, where cij is the capacity of the edge eij, and vi represents any node on $\mathrm{P}$, while vd represents the destination or target node.

The node vi has the following proprieties:

- Each node vi has a cost congestion cc such that:

$$
c c\left(v_{i}\right)=\frac{N p \times T_{\max }}{V p}
$$

where $\mathrm{Vp} \geq 1$ and $\mathrm{Np}$ is the total number of flow (packets) in the network, Tmax is the maximum token offered to each node vi, $\mathrm{Vp}$ is the number of packets visited the node vi.

- The updated edge cost of the path from vi to vd can be computed as follow:

$$
S v i=w i c c(v i)
$$

- $\quad$ The cost of all paths of vi can be presented as: Svi $=\{$ Svi1, Svi2, Svi3... Svik $\}$, where $\mathrm{k}=$ number of all paths of vi. 


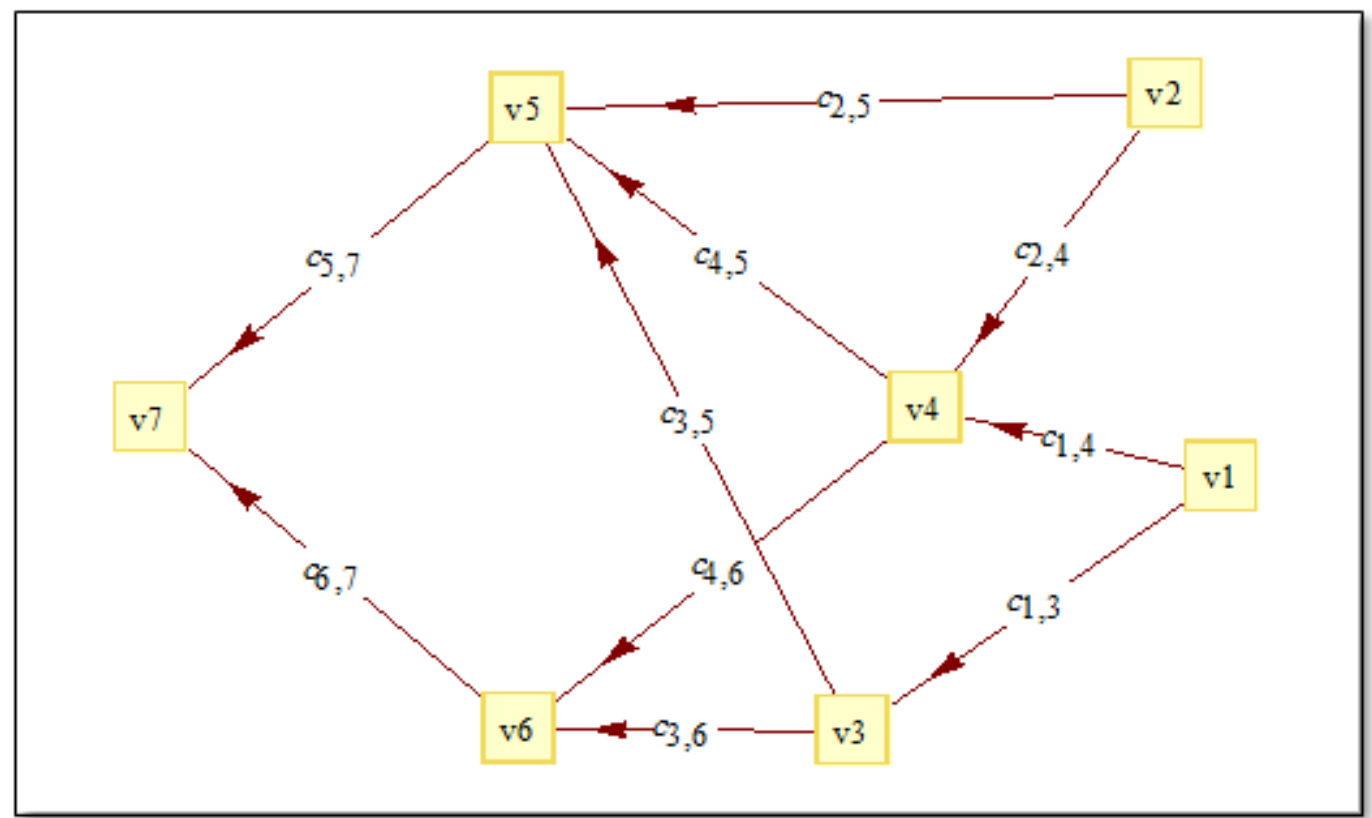

- $\quad$ The mixed strategies model (random scheme) can be illustrated as follows: $P_{v i}^{m}=$ the player vi has a profile of $\mathrm{m}$ strategies (probability profile), $P_{v i}^{k}=\left\{p_{v i}^{1}, p_{v i}^{2}, p_{v i}^{3}, \ldots, p_{v i}^{m}\right\}$, such that $p_{v i}^{k} \geq 0$ for each strategy $\mathrm{k}$, and $\sum_{k=1}^{m} p_{v i}^{k}=1, P_{v j}^{n}=$ the player vj has a profile of $\mathrm{n}$ strategies (probability profile), $P_{v j}^{Q}=\left\{p_{v j}^{1}, p_{v j}^{2}, p_{v j}^{3}, \ldots, p_{v j}^{n}\right\}$, such that $p_{v j}^{Q} \geq 0$ for each strategy $\mathrm{Q}$, and $\sum_{Q=1}^{m} p_{v i}^{Q}=1$.

- The players' payoff functions are:

$v_{i}^{\pi}=\sum_{r=1}^{n} \sum_{c=1}^{m} p_{v}^{i} p_{v}^{j} s_{r c}^{v i}$

$v_{j}^{\pi}=\sum_{r=1}^{n} \sum_{c=1}^{m} p_{v}^{i} p_{v}^{j} s_{r c}^{v j}$

where $S_{i j}^{v i}$ and $S_{i j}^{v j}$ are the cost of paths obtained by players vi and vj respectively. 


\section{QUANTAL RESPONSE EQUILIBRIUM (QRE)}

To study the players behaviors and determine whether they have noisy decisions, the Quantal Response Equilibrium (QRE) can be used to calculate equilibrium points. A QRE is a generalizing form of Nash equilibrium but with the discrete strategies. A Quantal response function maps the vector of expected payoffs from available choices into a vector of choice probabilities that is monotone in the expected payoffs. The results of QRE may differ from the Nash Equilibrium results because we assume the players may make error in their decisions to choose the pure strategies. The QRE based on Logic Equilibrium which has the following formula:

$p_{i}=\frac{e^{\lambda\left(E U_{i}\right)}}{\sum_{i=1}^{N} e^{\lambda\left(E U_{i}\right)}}$

$$
E U_{i}=\sum_{k=1}^{M}\left(q_{k} U_{i k}\right)
$$

where, pi is the probability of player 1 that choose strategy I out of $\mathrm{N}, \mathrm{q}_{\mathrm{k}}$ is other player probability of strategy $k$ out of M. $\lambda$ is a random variable. $U_{i k}$ the utility function of strategy $i$ and $k$ are chosen.

\section{The Proposed Algorithm (Game Traffic Route - GTR)}

The GTR based on the idea that any given graph G has many sub-graphs, and each sub-graph has two or more players. Each player has a profile of strategies (probability profile) to play against the others. For each sub-graph, the algorithm tries to find the maximum payoff of each player in an iterative way by adjusting the cost of link from source to destination of each path in sub-graph. The following algorithm illustrates the steps to find the maximum payoff of each player.

\section{RESULTS}

For evaluation purpose and to test our model, let consider the sub-graph which has initial weights that represent travel cost between edges from source to destination. The graph presents two players vi and vj that have two strategies of each of them to reach the destination vd. We can represent the travel cost by using a bimatrix, such as:

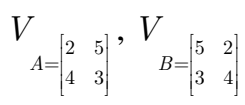

and by computing the $v_{i}^{\pi}$ and $v_{j}^{\pi}$ payoff function we can get:

$$
\begin{aligned}
& v_{i}^{\pi}=-4 p_{v_{i}}^{1} p_{v_{j}}^{1}+2 p_{v_{i}}^{1}+p_{v_{j}}^{1}+3 \\
& v_{j}^{\pi}=4 p_{v_{i}}^{1} p_{v_{j}}^{1}-2 p_{v_{i}}^{1}-p_{v_{j}}^{1}+4 \\
& \frac{\partial v_{i}^{\pi}}{\partial p_{v i}^{1}}=-4 p_{v j}^{1}+2 \rightarrow p_{v j}^{1}=\frac{1}{2}
\end{aligned}
$$




\section{GTR Algorithm}

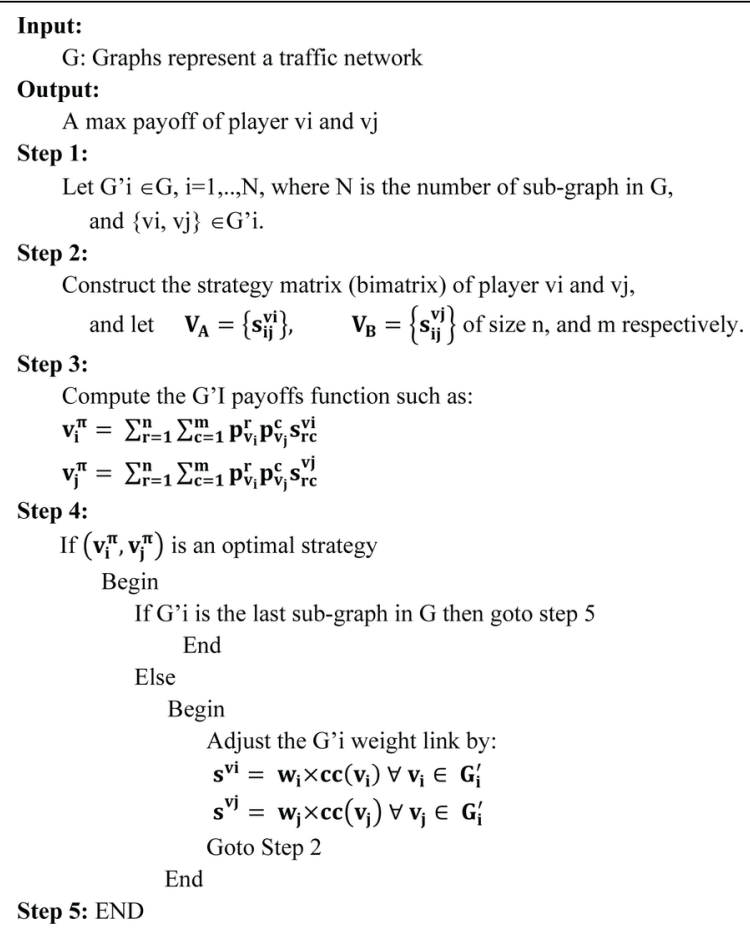

$\frac{\partial v_{j}^{\pi}}{\partial p_{v j}^{1}}=4 p_{v i}^{1}-1 \rightarrow p_{v i}^{1}=\frac{1}{4}$

Therefore, $v_{i}^{\pi}=\left(\frac{1}{4}, \frac{3}{4}\right)$ and $v_{j}^{\pi}=\left(\frac{1}{2}, \frac{1}{2}\right)$ is a mixed strategies equilibrium.

From Figure 2 and Table 1, the red curve of vi evolves till reaching a steady state at points $(0.25$, $0.75)$ which is equal to the optimal value achieved through Nash equilibrium with mixed strategy. Whereas, the blue curve of vj starts form an initial point $(0.5)$ and return to the same point, and that indicates the vj has only 0.5 probability over vi to strategies.

\section{CONCLUSION}

Choosing the best routing strategy is a great impact in network traffic routing researches. By adopting a special non cooperative game theoretic model, users (players) have the ability to present their strategies associated with preferred paths and recognize the one which has higher payoff. This research presents a mixed strategy game theory model for network routing problem. In addition, the QRE that verify the equilibrium has also presented and compared with mixed strategy equilibrium. The results show that the presented model has stated clearly there is equilibrium with mixed strategy of a given network. 
Figure 2. QRE of vi and vj

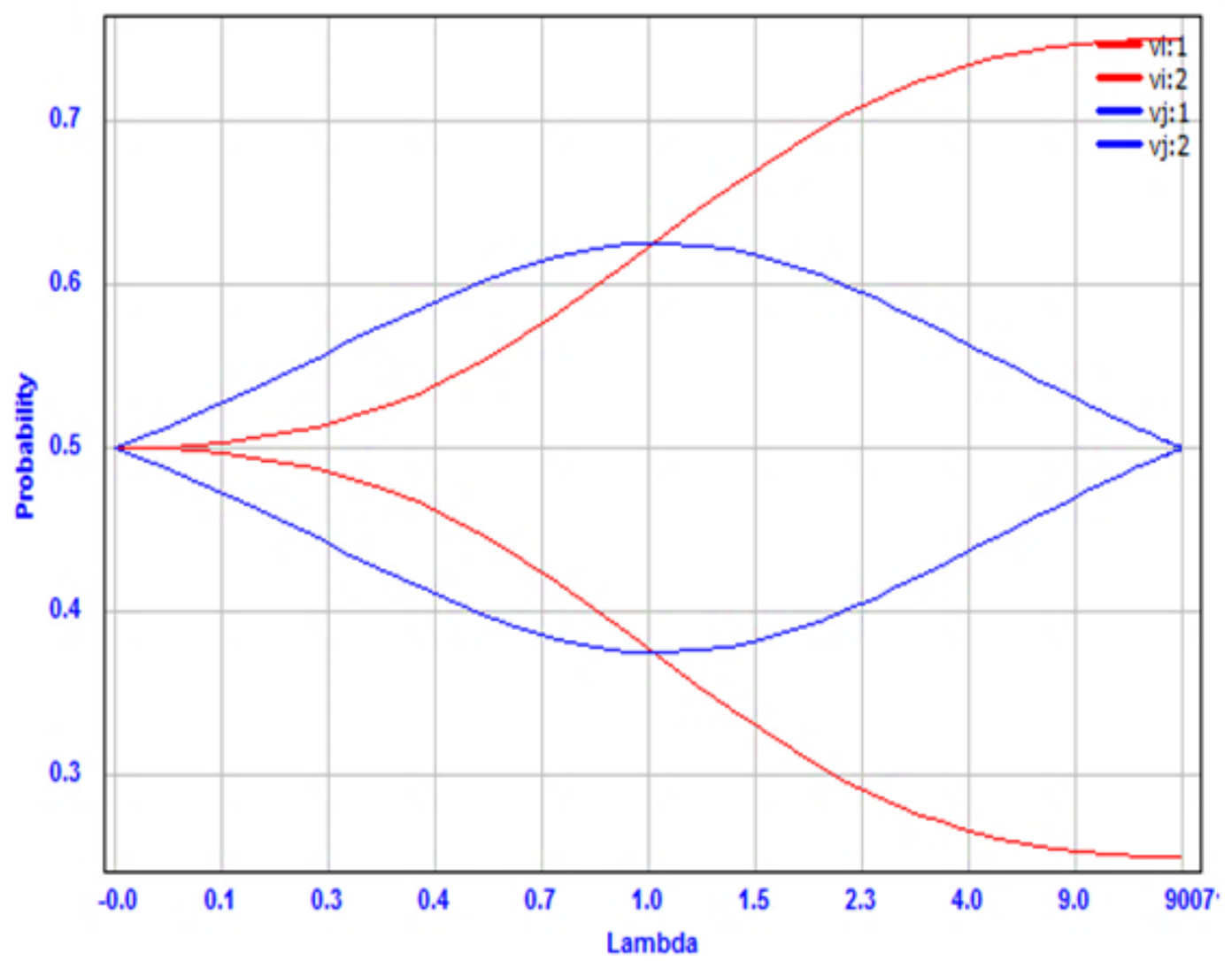

\section{Suggestions}

Evolutionary Game Theory (EGT) approach could be the next step of improving this work. By applying discrete replicator dynamic, an application of EGT, participants (users) with special behavior can be recognized among others over time. Hence, EGT provides participants away of pursuing better strategies with a higher payoff.

\section{ACKNOWLEDGMENT}

Many grateful thanks to Al-Mustansiriyah University Teaching Staff, Baghdad, Iraq, and Email: https://uomustansiriyah.edu.iq, for its unlimited support to achieve this work. 
Table 1. A snapshot of QRE data

\begin{tabular}{|c|c|c|c|c|c|}
\hline$\#$ & & $p_{v i}^{1}$ & $\left(\mathbf{1}-p_{v i}^{1}\right)$ & $p_{v j}^{1}$ & $\left(1-p_{v j}^{1}\right)$ \\
\hline 1 & 0 & 0.5 & 0.5 & 0.5 & 0.5 \\
\hline 2 & 0.0244 & 0.49985 & 0.50015 & 0.506118 & 0.493882 \\
\hline 3 & 0.0514 & 0.49934 & 0.50066 & 0.512822 & 0.487178 \\
\hline 4 & 0.0811 & 0.498367 & 0.501633 & 0.520132 & 0.479868 \\
\hline 5 & 0.1137 & 0.496808 & 0.503192 & 0.528052 & 0.471948 \\
\hline 6 & 0.1498 & 0.494523 & 0.505477 & 0.536565 & 0.463435 \\
\hline 7 & 0.1895 & 0.491352 & 0.508648 & 0.545624 & 0.454376 \\
\hline 8 & 0.2334 & 0.487128 & 0.512872 & 0.555141 & 0.444859 \\
\hline 9 & 0.2820 & 0.481676 & 0.518324 & 0.564986 & 0.435014 \\
\hline 10 & 0.3359 & 0.474833 & 0.525167 & 0.57497 & 0.42503 \\
\hline : & : & : & : & : & : \\
\hline : & : & : & : & : & : \\
\hline 154 & 5872.1 & 0.25 & 0.75 & 0.5 & 0.5 \\
\hline 155 & 6459.2 & 0.25 & 0.75 & 0.5 & 0.5 \\
\hline 156 & 7105.7 & 0.25 & 0.75 & 0.5 & 0.5 \\
\hline 157 & 7816.7 & 0.25 & 0.75 & 0.5 & 0.5 \\
\hline 158 & 8597.2 & 0.25 & 0.75 & 0.5 & 0.5 \\
\hline 159 & 9457.8 & 0.25 & 0.75 & 0.5 & 0.5 \\
\hline 160 & 1040.6 & 0.25 & 0.75 & 0.5 & 0.5 \\
\hline
\end{tabular}




\section{REFERENCES}

Allen, B. M., \& Luiz, A. D. (2006). Game theory for wireless engineers. Morgan \& Claypool.

Andra, G., Jozsef, J. B., Attila, K., Gabor, R., \& Dmitri, K. (2015). Navigable networks as nash equilibria of navigation games. Nature Communications. PMID:26138277

B., Y. (2019). Research on Regional Traffic Coordination Optimization Control Based on Game Theory. In IEEE 3rd Information Technology, Networking, Electronic and Automation Control Conference (ITNEC) (pp. 2427-2431). Chengdu, China: IEEE.

D., E., \& J., K. (2010). Reasoning about a highly connected world. Cambridge University Press.

Hyunmyung, K., Jun-Seok, O., \& R, J. (2009). Effect of user equilibrium assumption on network traffic pattern. KSCF Journal of Civil Engineering, 117-127.

J., L., J., L., Q., Y., \& B., C. (2019). A Multi-Vehicle Cooperative Routing Method Based on Evolutionary Game Theory. In IEEE Intelligent Transportation Systems Conference (ITSC) (pp. 987-994). Auckland, New Zealand: IEEE.

Jing, Y. S., Wei, Y. L., \& Kun, Y. (2009). A network routing algorithm based on the coalitional game theory. International Conference on Computational Intelligence and Natural Computing. doi:10.1109/CINC.2009.252

Ken, B. (2008). Game Theory A Very Short Introduction. Academic Press.

L., H., Y., X., L., Y., Z., J., \& C., N. (2010). Combined Traffic Assignment Model with Game Theory. International Conference on Intelligent Computation Technology and Automation, 978-981.

Michael, M., Eilon, S., \& Shmuel, Z. (2013). Game theory. Cambridge University Press.

Mohammed, E., Ahmed, A. E., \& Abdallah, A. H. (2015). An intersection game-theory-based traffic control algorithm in a connected vehicle environment. Researchgate. www.researchgate.net/publication/308812919

Nan, L., Ilya, K., Anouck, G., \& Yildiray, Y. (June 2018). Game theoretic modeling of vehicle interactions at unsignalized intersections and application to autonomous vehicle control. Annual American Control Conference $(A C C)$.

Noam, N., Tim, R., Eva, T., \& Vijay, V. (2007). Algorithmic game theory. Cambridge University Press.

Prisner, E. (2014). Game Theory Through Examples. Mathematical Association of America MAA. 
Ammar Kamel received his first M.Sc. in computer science from King Abdullah II School for Information Technology (KASIT), Jordan University, Jordan, in 2003, and a second M.Sc. degree was received from Western Michigan University in 2012. Also, he received his Ph.D. from Western Michigan University (WMU) in 2013. Before joining in the Ph.D. program, Dr. Ammar was an active teacher and researcher in the Computer Department at KASIT. His research interests include Network Management, Cloud Computing, and web services development and analysis. when he was a Ph.D. student at WMU, Dr. Ammar was a team member of the Cisco project to develop new techniques for network failure prediction. MS.c. 2004, Academic title: Lecturer 2004. Papers: 02, Administrative position: Head of the Computer \& Internet Department: 3 years.

Zaid Shafeeq Bakr is an engineer and Assistant Lecturer in Al-Nahrain University / college of information engineering. He has hold 3 years of university-level teaching experience in computer science, communication and network technologies and has a good skills in software engineering including cloud computing infrastructure, SAN storage, virtualization, IoT, database management, web site design, and Python programming. In 2008, he got his B.Sc in information engineering field from Al-nahrain University in Iraq, then in 2017, he got his M.Sc. in Networking Engineering and Internet Technologies field from Al-nahrain University in Iraq. Specialization: Digital Image Processing, Ph.D. 2006, Academic title: Professor 2015. Books: 30, Papers: 80, Supervision of postgraduate students: 30. International conferences: 15. Administrative position: Head of the Computer Science Department: 7 years. 